\title{
Optimising Post-Operative Discharge Analgesia For Patients Undergoing Major Shoulder Surgery (Rotator Cuff Repair Or Shoulder Replacement)
}

Dr Liam Sheppard (CT1 Anaesthetics, Royal Blackburn Hospital),

Dr Renee Ford (Consultant Anaesthetist, Queen Alexandra Hospital)

\section{1) Introduction}

After the effects of the nerve blockade have worn off, shoulder surgery patients can have moderate to severe pain for more than 48 hours after surgery ${ }^{1}$. A pragmatic approach to address post operative pain is through diligent use of simple oral analgesics combined with weak and strong oral opioids. There is currently no widely adopted guideline for the discharge analgesia for this group of patients at Queen Alexandra Hospital.

\section{2) Aims}

- Identify variability in prescribing practice for post operative analgesia on discharge for major shoulder surgery (MSS) patients.

- See if a difference exists between week day and weekend discharges regarding discharge analgesia provided.

- Measure the impact of the aforementioned prescribing variability on patient's pain scores and need to access medical services for additional analgesia.

\section{3) Methods}

Patient's consent gained for telephone follow up.

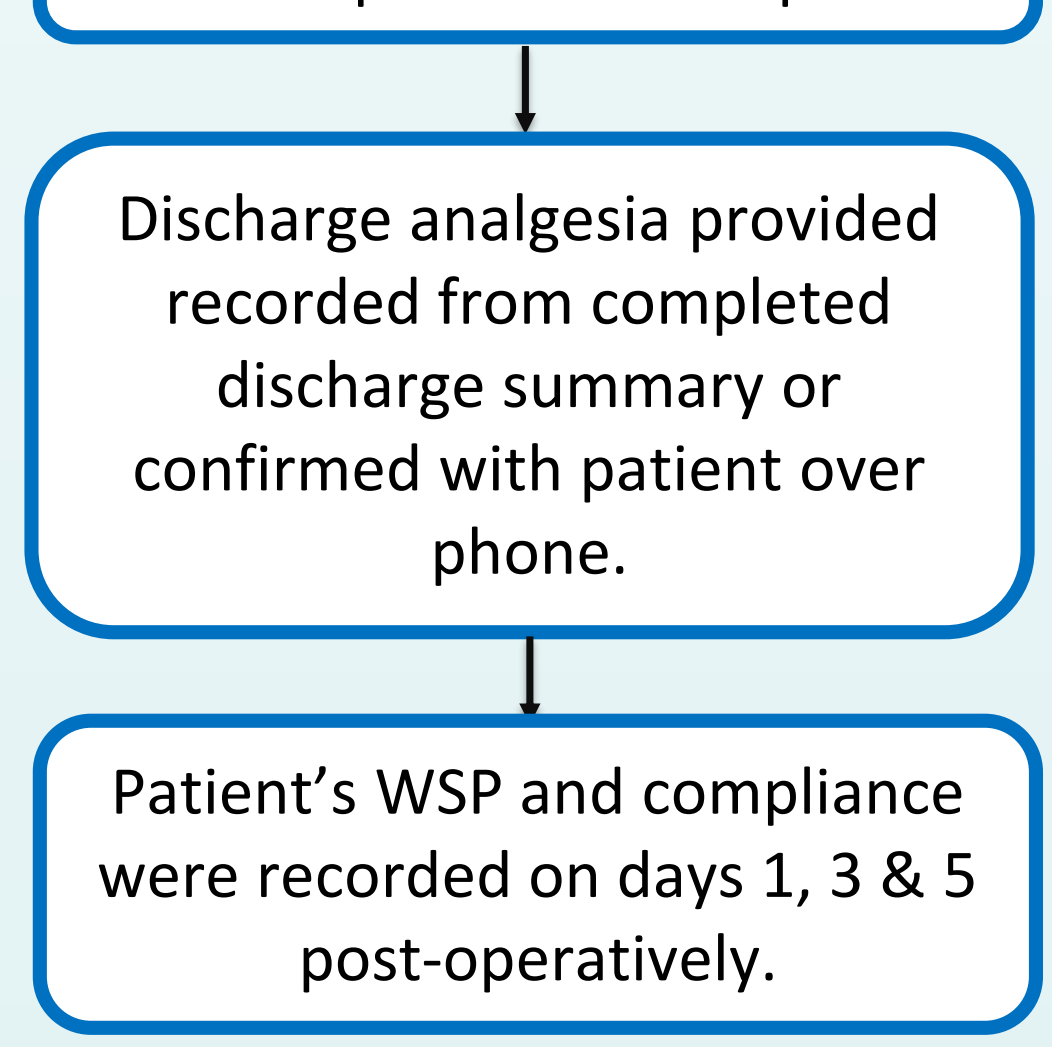

\section{* Wessex Pain Score}

- $\mathbf{0}=$ no pain at rest or on movement

- $\quad \mathbf{1}=$ no pain at rest, mild pain on movement

- $\mathbf{2}$ = moderate pain at rest or on movement

- $\mathbf{3}$ = severe pain at rest

\section{4) Key Results \& Outcomes}

20 patients were followed up, 10 weekend \& 10 weekday discharges.

The average 5-day pain score for weekend patients was $\underline{\mathbf{2 . 1 6 5}}$, vs 1.866 for weekday patients.

The 5-day average pain scores for weekend vs. weekday shoulder replacements were 2.61 vs. 1.722
All but one patient was discharged with at least 1 simple analgesic and 1 weak opiate

At day 5 post-op, 8/10 vs. 4/10 weekday to weekend patients respectively were still using at least one weak opioid.

TWO patients required additional analgesia, both WEEKEND discharges.

\section{Weekday Discharge WPS vS. Weekend Discharge WPS}

\begin{tabular}{|lll|llll} 
Day 1 & Day 3 & Day 5 & & Day 1 & Day 3 & Day 5 \\
2 & 1 & 1 & 1 & 3 & 2 \\
0 & 2 & 2 & 3 & 3 & 3 \\
2 & 2 & 2 & 3 & 3 & 3 \\
3 & 2 & 2 & 1 & 3 & 3 \\
2 & 2 & 1 & 1 & 2 & 3 \\
\hline 3 & 2 & 2 & 3 & 3 & 3 \\
\hline 1 & 1 & 2 & 2 & 3 n/a & \\
\hline 3 & 3 & 3 & 2 & 2 & 1 \\
\hline 2 & 2 & 1 & 2 & 2 & 2 \\
2 & 1 & 2 & 1 & 1 & 1
\end{tabular}

\section{5) Conclusion}

- Weekend discharges of MSS patient at Queen Alexandra Hospital appear to experience more post-operative pain, especially shoulder replacements.

- To reduce unwarranted variation in discharge analgesia and improve overall pain management, a post-operative analgesia timetable specific to MSS patients will be developed. 